\title{
Detection of phospholipid oxidation in oxidatively stressed cells by reversed-phase HPLC coupled with positive-ionization electroscopy MS
}

\author{
Corinne M. SPICKETT*, Nicola RENNIE†, Helen WINTER $\dagger$, Laura ZAMBONIN†, Laura LANDI\$, Andreas JERLICH§, \\ R. Jörg SCHAUR $§$ and Andrew R. PITT†' \\ *Department of Immunology, University of Strathclyde, 27 Taylor Street, Glasgow G4 0NR, U.K., †Department of Pure and Applied Chemistry, University of Strathclyde, \\ Thomas Graham Building, 295 Cathedral Street, Glasgow G1 1XL, U.K., \$Department of Biochemistry, University of Bologna, Via Irnerio 48, 40126 Bologna, Italy, and \\ §Institute of Molecular Biology, Biochemistry and Microbiology, University of Graz, Schubertstraße 1, A-8010, Graz, Austria
}

\begin{abstract}
Measurement of lipid peroxidation is a commonly used method of detecting oxidative damage to biological tissues, but the most frequently used methods, including MS, measure breakdown products and are therefore indirect. We have coupled reversedphase HPLC with positive-ionization electrospray MS (LC-MS) to provide a method for separating and detecting intact oxidized phospholipids in oxidatively stressed mammalian cells without extensive sample preparation. The elution profile of phospholipid hydroperoxides and chlorohydrins was first characterized using individual phospholipids or a defined phospholipid mixture as a model system. The facility of detection of the oxidized species in complex mixtures was greatly improved compared with directinjection MS analysis, as they eluted earlier than the native lipids, owing to the decrease in hydrophobicity. In U937 and HL60 cells treated in vitro with t-butylhydroperoxide plus $\mathrm{Fe}^{2+}$, lipid oxidation could not be observed by direct injection, but LC-MS
\end{abstract}

allowed the detection of monohydroperoxides of palmitoyllinoleoyl and stearoyl-linoleoyl phosphatidylcholines. The levels of hydroperoxides observed in U937 cells were found to depend on the duration and severity of the oxidative stress. In cells treated with $\mathrm{HOCl}$, chlorohydrins of palmitoyloleoyl phosphatidylcholine were observed by LC-MS. The method was able to detect very small amounts of oxidized lipids compared with the levels of native lipids present. The membrane-lipid profiles of these cells were found to be quite resistant to damage until high concentrations of oxidants were used. This is the first report of direct detection by LC-MS of intact oxidized phospholipids induced in cultured cells subjected to oxidative stress.

Key words: chlorohydrin, electrospray ionization, HL60 cells, lipid peroxidation, U937 cells.

\section{INTRODUCTION}

The plasma membranes of most cells contain both polyunsaturated and monounsaturated lipids, which are susceptible to oxidative damage by free-radical processes or electrophilic addition reactions, e.g. reaction of hypochlorous acid $(\mathrm{HOCl})$ with a double bond. Such oxidative modification of the lipids is thought to lead to alterations in structure and fluidity of the membrane, affecting cell function, and can ultimately lead to loss of integrity [1,2]. Hence there is considerable interest in the detection and measurement of oxidative lipid damage, both in model systems and in many of the disease states that have now been shown to have an oxidative aetiology.

Methods for the analysis of oxidative damage to lipids differ considerably in the sensitivity and information offered, and can be divided essentially into two groups: those that measure initial products of oxidative attack, such as conjugated dienes and lipid hydroperoxides, and those that measure lipid-oxidation breakdown products, such as aldehydes. In the last decade GC-MS has emerged as a highly sensitive method for the analysis of aldehydes [3-5], fatty acids and their hydroperoxides [6], lipid hydroperoxides [7] and isoprostanes [8,9]. However, GC-MS has the disadvantage that extensive preparation of the sample is required to obtain a volatile form, and before phospholipids can be analysed they must first be hydrolysed to release the fatty acid, and hydroperoxides must be reduced to hydroxides, thus intro- ducing manipulation steps where information about the native lipid is lost. An alternative technique that is gaining in popularity is electrospray MS (ES-MS), a soft ionization technique which does not automatically result in fragmentation of the biomolecules [10], thus allowing analysis of complex mixtures. It also has the advantage that intact phospholipids can be detected directly without prior manipulation, thus offering a simple analysis without loss of information on the nature of the lipid $[11,12]$.

In terms of analysis of lipid oxidation in biological systems, most ES-MS studies have been carried out in negative-ion mode on oxidation products of fatty acids. There are reports on the detection of isoprostanes [13] and oxidized free fatty acids [14], including bromohydrins of mono- and polyunsaturated fatty acids [15]. An approach that has been used in a number of studies to detect oxidized fatty acids esterified in phospholipids is the initial separation of glycerophospholipid classes by normalphase HPLC, followed by base hydrolysis of the lipid and subsequent analysis of the fatty acid derivative by reversed-phase HPLC and negative-ion ES-MS. This has been used to investigate the oxidation of glycerophosphoethanolamine plasmalogens [16], and to analyse the formation of epoxyeicosanoic acids (EETs) and monohydroxyeicosanoic acids (HETEs) in redblood-cell membranes [17,18] and murine lung tissue [19]. Whereas this approach is informative and has generated very interesting information, it requires considerable sample mani-

Abbreviations used: ES-MS, electrospray MS; PC, phosphatidylcholine; RIC, reconstructed ion chromatogram; TIC, total ion current; t-BHP, t-butylhydroperoxide; EET, epoxyeicosanoic acid; HETE, monohydroxyeicosanoic acid; LC-MS, reversed-phase HPLC coupled with positive-ionization electrospray MS; GPC, glycerophosphatidylcholine.

1 To whom correspondence should be addressed (e-mail a.r.pitt@strath.ac.uk). 
pulation, and information on the intact phospholipid modification is lost, thus not exploiting some of the advantages of ES-MS.

Less research has been carried out using ES-MS for the analysis of intact phospholipid oxidation products. Recently we have reported the detection of hydroperoxides of intact phosphatidylcholines (PCs) by positive-ion ES-MS, following treatment of $\mathrm{PC}$ vesicles with t-butylhydroperoxide (t-BHP) and $\mathrm{Fe}^{2+}$ [20]. Depletion of native stearoylarachidonoyl PC was observed, with the concomitant appearance of molecular species at +32 , +64 and +96 a.m.u. (atomic mass units), corresponding to the addition of one, two and three molecules of dioxygen. Alternative oxidation products have also been observed by positive-ion ES-MS following the auto-oxidation of palmitoylarachidonoyl PC. Two major species were identified as the chainshortened products 1-palmitoyl-2-oxovaleryl-GPC (where GPC is glycerophosphatidylcholine) and 1-palmitoyl-2-glutarylGPC [21], and another major component was found to be 1palmitoyl-2-(5,6-epoxyisoprostane $\left.\mathrm{E}_{2}\right)$-GPC [22]. These oxidized phospholipids have been detected using ES-MS in oxidized lowdensity lipoprotein and atherosclerotic lesions in rabbits [21].

So far, very little work has been carried out on phospholipids from oxidatively stressed cells. Increased levels of HETEs and EETs from glycerophospholipids in red blood cells treated with t-BHP have been observed by ES-MS following base hydrolysis of the lipids, but there are no reports of direct detection of intact oxidized phospholipids in stressed cells. The direct detection of early products of lipid peroxidation in cells would offer a new approach to understanding cellular responses to oxidative membrane damage. We have investigated this and have found that whereas oxidized phospholipids could not be observed using direct-injection ES-MS analysis, coupling to reversed-phase HPLC allowed the detection of very low levels of intact phospholipid hydroperoxides and chlorohydrins from mammalian cells subjected to oxidative stress.

\section{EXPERIMENTAL}

\section{Materials}

The mammalian cell lines U937 and HL60 were from the European Collection of Animal Cell Cultures at Porton Down, Wilts., U.K. RPMI 1640 and fetal calf serum were obtained from Life Technologies, Inchinnan, Renfrewshire, Scotland, U.K. PCs and phosphatidylethanolamines were purchased from Sigma, Poole, Dorset, U.K. All solvents and chemicals used were of analytical quality.

\section{Preparation and treatment of vesicles}

Phospholipid vesicles were prepared from purified PCs as described previously [20] to give a final concentration of $10 \mathrm{mg} / \mathrm{ml}$ in water. For the formation of lipid hydroperoxides $10 \mu \mathrm{l}$ of vesicles were incubated aerobically overnight in the presence of $0.25 \mathrm{M}$ t-BHP and $0.1 \mathrm{mM} \mathrm{FeSO}_{4}$ in a total volume of $100 \mu \mathrm{l}$. Solutions of $\mathrm{FeSO}_{4}$ were prepared freshly immediately prior to use. For the formation of chlorohydrins $10 \mu \mathrm{l}$ of vesicles in $10 \mathrm{mM} \mathrm{NaH} \mathrm{PO}_{4}, \mathrm{pH} 6.0$, were incubated in the presence of $1.85 \mathrm{mM} \mathrm{NaOCl}$ in a total volume of $100 \mu \mathrm{l}$. The incubations were carried out at $\mathrm{pH} 6.0$ to ensure that the majority of the hypochlorite was in the protonated form, which is required for oxidative attack. A slightly acidic $\mathrm{pH}$ is moreover assumed to occur in the phagolysosome of phagocytes. Aliquots $(10 \mu \mathrm{l})$ were removed after $40 \mathrm{~min}$ and mixed with $90 \mu \mathrm{l}$ of methanol/hexane $(71: 5, \mathrm{v} / \mathrm{v})$; further dilutions were carried out in $71: 5: 7$ (by vol.) methanol/hexane/0.1 M ammonium acetate and analysed with- out further preparation by either ES-MS or reversed-phase HPLC coupled with positive-ionization electroscopy MS (LC-MS).

\section{Culture and preparation of mammalian cell lines}

The human monocytic cell line U937 was cultured in RPMI 1640 medium containing $25 \mathrm{mM}$ Hepes, $0.3 \mathrm{~g} / \mathrm{L}$-glutamine and $10 \%$ fetal calf serum, at $37{ }^{\circ} \mathrm{C}$ with $5 \% \mathrm{CO}_{2}$. The cells were subcultured approximately every $72 \mathrm{~h}$ by dilution to $3 \times 10^{5}$ cells $/ \mathrm{ml}$, and were allowed to grow to $\approx 1 \times 10^{6}$ cells $/ \mathrm{ml}$. The myeloid cell line HL60 was cultured in the same medium but was diluted to $2 \times 10^{5}$ cells $/ \mathrm{ml}$ for subculturing. For both cell types the viability was assessed by counting the cells in the presence of $0.25 \%$ Trypan Blue. Cells were harvested by centrifugation at $600 \mathrm{~g}$ for 10 min and washed twice in RPMI 1640 without fetal calf serum.

\section{Treatment of cells with oxidants}

Cells (0.2 g) were resuspended with $1 \mathrm{ml}$ of PBS (100 mM $\mathrm{Na}_{2} \mathrm{HPO}_{4}, 25 \mathrm{mM} \mathrm{NaH}_{2} \mathrm{PO}_{4}$ and $0.9 \%, \mathrm{w} / \mathrm{v}, \mathrm{NaCl}$ ), and $0.6 \mathrm{ml}$ aliquots were placed in $1.5 \mathrm{ml}$ reaction tubes before centrifuging at $600 \mathrm{~g}$ and removing the supernatant. Treatments were started by the addition of $0.5 \mathrm{ml}$ of oxidant solution in PBS, or PBS only (control). Experiments with $\mathrm{HOCl}$ were carried out in PBS at pH 6.0 and t-BHP was diluted in PBS, pH 7.4. For the initial oxidative treatments the oxidant solutions used were $3.5 \mathrm{mM}$ $\mathrm{HOCl}$ or $1 \mathrm{M} \mathrm{t}$-BHP with $1 \mathrm{mM} \mathrm{FeSO}$, and the cells were incubated for $30 \mathrm{~min}$ at $37^{\circ} \mathrm{C}$. For the dose-response experiments the t-BHP concentration was varied from $50 \mathrm{mM}$ to $1 \mathrm{M}$ with an incubation time of $30 \mathrm{~min}$, and time courses were $0-4 \mathrm{~h}$ with $0.5 \mathrm{M}$ t-BHP. All treatments were terminated by centrifuging at $10000 \mathrm{~g}$, and the cells were washed once in PBS to remove traces of the oxidants before extraction. Three experiments were carried out for each of the initial treatments, and two sets of data were obtained for the time-course and dose-response experiments; the results for both sets were found to be very comparable.

\section{Extraction of cell lipids}

The cells were extracted by a modification of the procedure of Bligh and Dyer [23]. To each tube containing the pelleted cells, $0.5 \mathrm{ml}$ of methanol at $50{ }^{\circ} \mathrm{C}$ was added, and the mixture was incubated in a sonicating water bath for $15 \mathrm{~min}$. An equal volume of chloroform was then added, and the sonication was repeated. Finally $0.5 \mathrm{ml}$ of $0.88 \% \mathrm{KCl}$ was added, and the mixture was vortexed well before centrifuging at $10000 \mathrm{~g}$ to separate the aqueous and organic layers. The organic layer was transferred to a clean tube and dried under a stream of nitrogen. The lipid extracts were stored at $-70{ }^{\circ} \mathrm{C}$ prior to analysis by ESMS or LC-MS.

\section{ES-MS and LC-MS}

ES-MS was performed in positive-ion mode on a VG Platform mass spectrometer (Micromass, Altrincham, Cheshire, U.K.). For direct infusion of samples the solvent system was 71:5:7 (by vol.) methanol/hexane $/ 0.1 \mathrm{M}$ ammonium acetate, with a flow rate of $20 \mu \mathrm{l} / \mathrm{min}$. The source temperature of the mass spectrometer was set to $75^{\circ} \mathrm{C}$, with a nebulizing gas flow of $20 \mathrm{l} / \mathrm{h}$ and a drying gas flow of $400 \mathrm{l} / \mathrm{h}$. Data were collected between 400 and $1000 \mathrm{~m} / z$ with a sweep time of $5 \mathrm{~s}$. The spectra were a combination of 10-12 consecutive scans, and after summing were mean-smoothed twice with a window of $0.3 \mathrm{Da}$.

LC-MS was carried out using an adaptation of the HPLC method of Kim et al. [24], with a Shimadzu LC-10 system and a 
Phenomenex Luna $\mathrm{C}_{8}$ column $(5 \mu \mathrm{m}$ RP-Sct, $1 \mathrm{~mm}$ inner diameter $\times 150 \mathrm{~mm})$. This column was operated at a flow rate of $100 \mu \mathrm{l} / \mathrm{min}$ with an isocratic solvent system of 71:5:7 (by vol.) methanol/hexane/0.1 M ammonium acetate. It was important to use freshly prepared solvent to prevent changes in retention time that occurred over time due to differential evaporation of the solvent components.

Cell extracts were reconstituted in 1:5 chloroform/methanol (usually $100 \mu \mathrm{l}$ total volume) and diluted 10-fold in running solvent; $10 \mu \mathrm{l}$ of sample was injected per run. The eluent from the column was split $\approx 1: 1$ between the mass spectrometer and waste. Peak-top spectra were collected usually between 650 and $950 \mathrm{~m} / \mathrm{z}$ with a sweep time of $2 \mathrm{~s}$. Extracted mass chromatograms showing individual molecular species were generated using MassLynx software (Micromass), and were mean-smoothed as described above. In all the spectra and chromatograms shown the percentage scale on the vertical axes corresponds to intensity related to that of the largest peak in the region analysed, unless otherwise stated.

Peak areas were estimated for the time-course and doseresponse experiments by cutting and weighing the relevant peaks in the printed chromatograms, and the units quoted are therefore mg. The expected isotopic mass ratios for phospholipids containing chlorine were calculated using Chemputer software (Sheffield, U.K.).

\section{RESULTS}

\section{Cell phospholipid separation by LC-MS}

Figure 1 shows the separation of a selection of phospholipids extracted from control-cultured U937 cells and their observation by LC-MS, including the total ion current (TIC) and reconstructed ion chromatograms (RICs) at specific mass-to-charge ratios $(m / z)$. In most cases, the peaks in the RICs are expected to contain a single major component at the mass indicated, although a number of phospholipids could give the same mass ion. In positive-ion mode the strongest species observed are PCs or sphingomyelins, due to the positive charge on the choline headgroup; PCs are a major component of most mammalian cell membranes. The phospholipids were identified by comparison with previously published spectra $[11,12,20]$, bearing in mind the limitations described therein for single-analysis MS [20]. These include the inability to differentiate double unsaturation in one chain from mono-unsaturation in both and, for example, two $\mathrm{C}_{16}$ chains from one $\mathrm{C}_{18}$ and one $\mathrm{C}_{14}$. Suggested assignments of major species are based on the previously published distributions of fatty acids for mammalian PCs, but identification using the total number of carbons in the fatty acyl chains and double bonds will also be given (e.g. $\mathrm{C}_{32: 1}$ ).

Cultured U937 cells were found to contain a variety of phospholipids, with the major components containing fatty acids with chains of 14-18 carbons. Longer-chain highly unsaturated fatty acids, including arachidonic acid, were present only as minor components, probably reflecting the fact that the cells were undifferentiated [25]. The majority of the phospholipids were found to occur in the protonated, rather than sodiated, form using this methodology, and it can be seen that separation occurred according to the headgroup, fatty acyl chain length and degree of unsaturation. For a given chain length, lipids containing more unsaturated fatty acids eluted earlier from the column: for example, $\mathrm{C}_{34: 2} \mathrm{PC}$ (e.g. palmitoyl-linoleoyl PC) at $758 \mathrm{~m} / \mathrm{z}$ eluted earlier (11.9 min) than $\mathrm{C}_{34: 1} \mathrm{PC}$ (e.g. palmitoyloleoyl PC) at $760 \mathrm{~m} / z$ (15.0 min). Increased chain length of the fatty acids resulted in longer retention times for the lipids, as can be seen by comparing the elutions of $\mathrm{C}_{30: 0}$ PC (e.g. myristoylpalmitoyl PC) with a mass of 706 at 10.0 min and $\mathrm{C}_{32: 0} \mathrm{PC}$ (e.g. dipalmitoyl PC) with a mass of 734 at $13.75 \mathrm{~min}$. Some extra peaks are visible in the chromatograms due to the isotopic spill-over of the double ${ }^{13} \mathrm{C}$-isotope peak of phospholipids at $2 \mathrm{~m} / z$ smaller, i.e. containing one more double bond. This is apparent in the extracted ion chromatogram at $734 \mathrm{~m} / \mathrm{z}$ where a double isotope peak from $\mathrm{C}_{32: 1} \mathrm{PC}(732 \mathrm{~m} / \mathrm{z})$ appears at $10.91 \mathrm{~min}$, and in the $788 \mathrm{~m} / \mathrm{z}$ trace at 16.34 min corresponding to $\mathrm{C}_{36: 2} \mathrm{PC}(786 \mathrm{~m} / \mathrm{z})$. This emphasizes the value of LC-MS, as the contribution of different species with the same molecular mass can be distinguished, in contrast to analysis by direct-injection ES-MS. Components with molecular masses corresponding to phosphatidylethanolamines were also observed, and were found to elute earlier than the equivalent PCs (results not shown).

\section{Observation of oxidatively modified phospholipids in model systems}

In order to characterize the elution profiles of oxidatively modified phospholipids, phospholipid vesicles were treated with oxidants to provide a simple model system. Figure 2 shows the results for vesicles treated with the radical oxidizing system t-BHP and $\mathrm{Fe}^{2+}$ overnight in the presence of air. The TIC chromatogram shows a variety of oxidized phospholipids eluting in the first $3-10 \mathrm{~min}$, and the native lipids eluting at $12 \mathrm{~min}$ onwards. The UV absorbance at $234 \mathrm{~nm}$ was also monitored, and although rather weak it supported the identification of these early components as conjugated dienes (results not shown). In the right-hand panel, the RICs at 842, 874 and $906 \mathrm{~m} / \mathrm{z}$ show the appearance of products corresponding to the addition of onethree dioxygens to $\mathrm{C}_{38: 4} \mathrm{PC}$ (stearoylarachidonoyl PC, $810 \mathrm{~m} / z$ ). The trishydroperoxide eluted earliest at $3.2 \mathrm{~min}$, followed by bishydroperoxides at 4-5 min with the mono-hydroperoxides eluting last at 8.6 and $9.76 \mathrm{~min}$; this would be expected owing to their increasing hydrophobicity. For both the mono- and bishydroperoxides several components were partially resolved, which are thought to correspond to isomers resulting from oxidation at different sites in the arachidonoyl chain. In the lefthand panel, the signals at $790 \mathrm{~m} / \mathrm{z}(4.9 \mathrm{~min})$ and $818 \mathrm{~m} / \mathrm{z}$ $(6.7 \mathrm{~min})$ are assigned to the monohydroperoxides of $\mathrm{C}_{34: 2}$ (native mass 758) and $\mathrm{C}_{36: 2}$ (native mass 786). Each of the components assigned as hydroperoxides showed two sourceinduced breakdown products with losses of 18 and 34 mass units, corresponding to dehydration or the loss of hydrogen peroxide; this provided support for the assignment as hydroperoxide [14]. None of the signals assigned to phospholipid hydroperoxides were observed in control vesicles.

In addition to lipid peroxidation, the effect of $\mathrm{HOCl}$ on phospholipid vesicles was investigated. The formation of chlorohydrins can be detected by ES-MS as an increase in mass of 52, corresponding to the addition of $\mathrm{HOCl}$ across a double bond [26]. Figure 3(a) shows the TIC and RICs of phospholipid vesicles treated with $1.85 \mathrm{mM} \mathrm{HOCl}$ for $40 \mathrm{~min}$, demonstrating the appearance of components with an increase in mass of 52 following incubation with $\mathrm{HOCl}$, which elute significantly earlier in the chromatogram than the native lipids. In the RIC at $810 \mathrm{~m} / \mathrm{z}$, the large peak at $17.7 \mathrm{~min}$ corresponds to native stearoylarachidonoyl PC $\left(\mathrm{C}_{38: 4} \mathrm{PC}\right)$, while the much smaller signal eluting at $5.0 \mathrm{~min}$ was assigned as a monochlorohydrin of $\mathrm{C}_{34: 2} \mathrm{PC}$ (native mass 758). The strong signal at $5.9 \mathrm{~min}$ in the $812 \mathrm{~m} / z$ RIC was assigned as the monochlorohydrin of $\mathrm{C}_{34: 1} \mathrm{PC}$, which accumulates to higher levels as it cannot undergo further reaction with $\mathrm{HOCl}$. The RIC at $838 \mathrm{~m} / z$ shows the appearance of two monochlorohydrin isomers of $\mathrm{C}_{36: 2} \mathrm{PC}$ at 6.26 and $6.96 \mathrm{~min}$, with a minor native phospholipid of the same mass at 

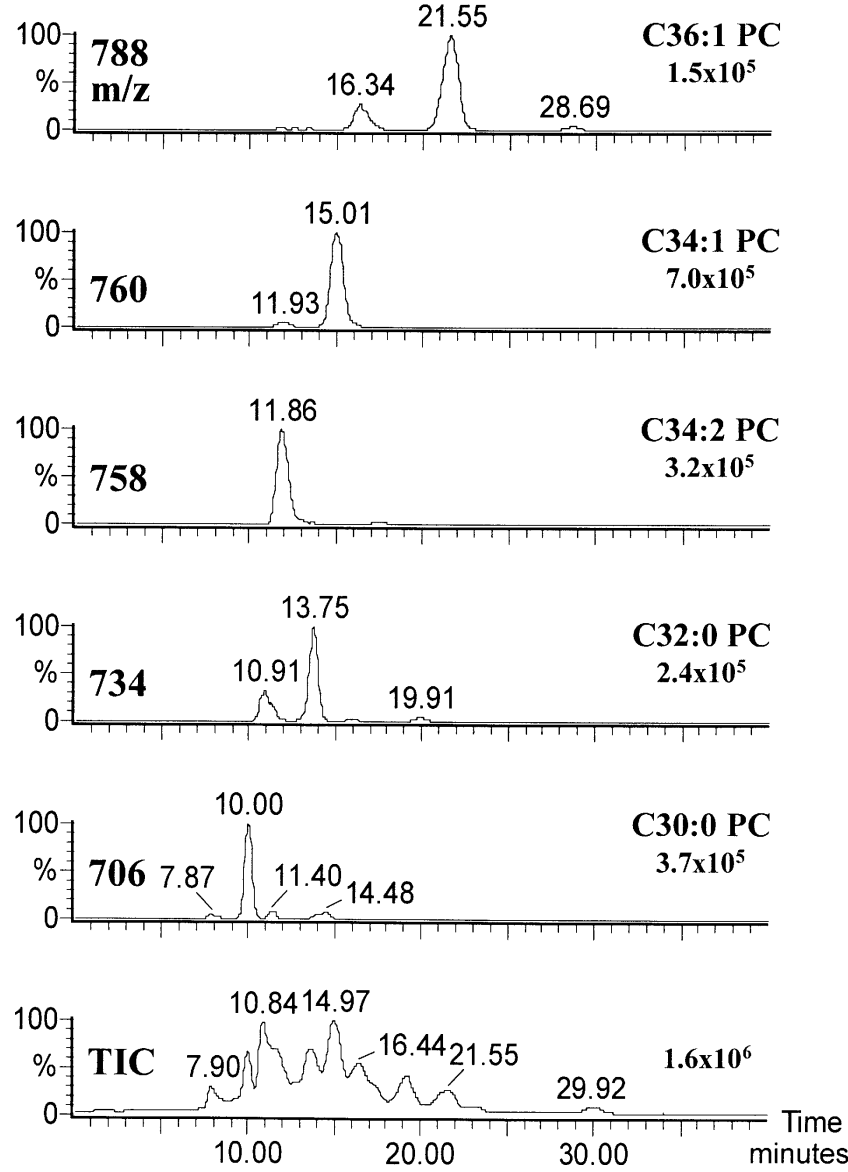

\section{Figure 1 LC-MS chromatograms of PCs from U937 cells}

The cell lipids were extracted in chloroform/methanol and dried under nitrogen. The extract was reconstituted in LC solvent (71:5:7 methanol/hexane/0.1 M ammonium acetate) and LC was carried out using a Phenomenex Luna column with a flow rate of $0.1 \mathrm{ml} / \mathrm{min}$. Peak-top data were collected between 650 and $950 \mathrm{~m} / \mathrm{z}$ with a sweep time of $2 \mathrm{~s}$. Total ion current (TIC) and reconstructed ion chromatograms (RICs) showing individual molecular species are shown; RICs were generated using MassLynx software and are mean-smoothed. $100 \%$ on the vertical axes corresponds to the ion current intensity indicated on the right-hand side of the chromatograms, below the lipid assignment. The vertical axes of the chromatograms are not linked, to allow observation of minor components.

$14.9 \mathrm{~min}$. The RIC at $862 \mathrm{~m} / \mathrm{z}$ shows the formation of several monochlorohydrins of $\mathrm{C}_{38: 4}$ PC eluting between 6.7 and $9.86 \mathrm{~min}$, while the peak at 3.04 min was identified as a bischlorohydrin of $\mathrm{C}_{34: 2} \mathrm{PC}$, based on the isotopic distribution pattern. Co-eluting with the modified lipids at $862 \mathrm{~m} / z$ were other components at $864 \mathrm{~m} / z$, assigned as the ${ }^{37} \mathrm{Cl}$ isotopes of the chlorohydrins. This can be seen more clearly in Figure 3(b), which shows the summed spectrum corresponding to the part of the chromatogram where the modified lipids eluted. This provided additional support for the identification of these components as chlorohydrins. For example, the ratio of $862: 864$ closely matched the calculated mass distribution for the presence of chlorine in this molecule (100:49). Similarly, the monochlorohydrin at $812 \mathrm{~m} / \mathrm{z}$ and bischlorohydrin at $914 \mathrm{~m} / z$ show ${ }^{37} \mathrm{Cl}$ isotope peaks with the appropriate isotope distribution. Details of the oxidative modifications of phospholipids observed most commonly are summarized in Table 1.

The detection limit for PCs using this method was found to be $\approx 200 \mathrm{fmol}$, which was comparable with the detection limit for
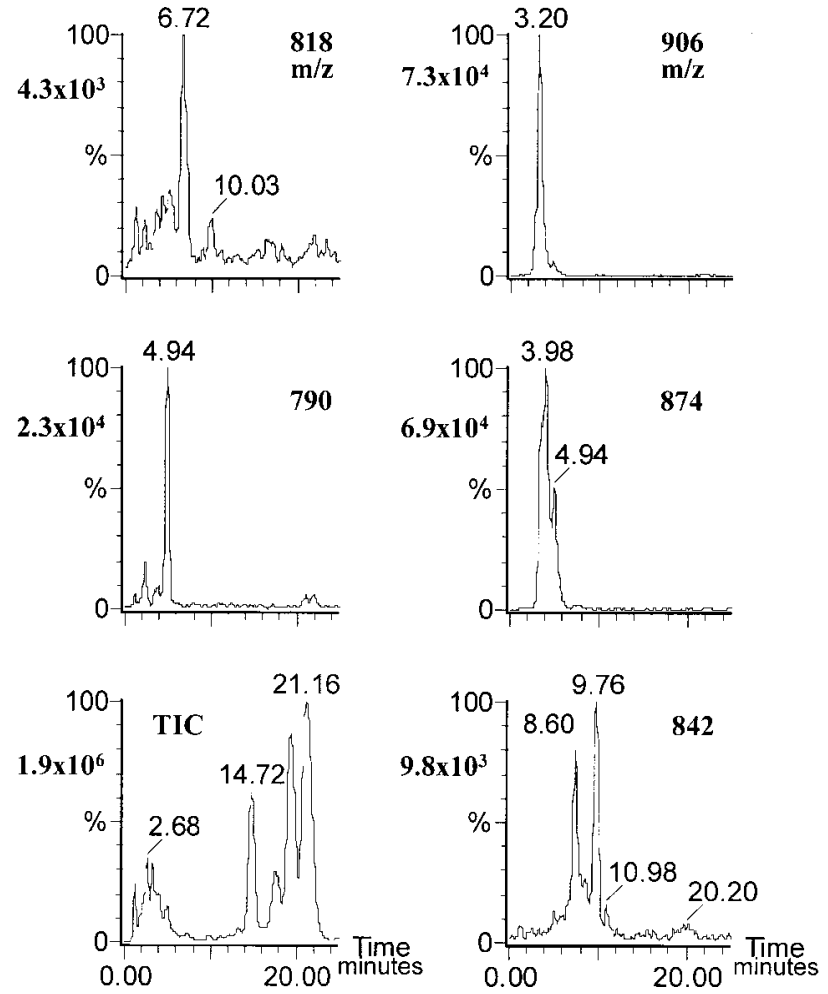

Figure 2 The detection of hydroperoxides in phospholipid vesicles

The TIC of phospholipid vesicles treated overnight with t-BHP and $\mathrm{Fe}^{2+}$ and RICs at five different masses $(\mathrm{m} / \mathrm{z})$ are shown. The LC-MS was carried out as described for Figure 1, except that the peak-top data were collected between 700 and $1000 \mathrm{~m} / \mathrm{z} .100 \%$ on the vertical axes corresponds to the ion current intensity indicated next to the $y$ axis of the chromatograms; the vertical axes are not linked.

direct infusion of an individual lipid species. However, the use of LC-MS greatly facilitated the detection of oxidized species in complex lipid mixtures: with direct infusion minor species were swamped in the spectrum by components with stronger signals, and it was not possible to differentiate intact oxidized lipids from native lipids with the same molecular mass.

\section{Observation of modified phospholipids from oxidatively stressed cells}

The main aim of this work was to investigate whether intact phospholipid hydroperoxides and chlorohydrins could be observed in oxidatively stressed cells using electrospray detection, as this allows the simultaneous observation of a variety of different oxidized lipids, thus offering more information about the events occurring in oxidative stress. In preliminary work using direct injection it was found to be impossible to detect the formation of such species in these cultured cell lines (results not shown). There was no evidence for the formation of chlorohydrins or hydroperoxides, and few changes in the lipid profile were observed, indicating that there had been no significant modification of the lipids, despite the relative severity of the treatments imposed.

When U937 or HL60 cells subjected to comparable treatments with t-BHP plus $\mathrm{Fe}^{2+}$ were extracted and analysed by LC-MS, 

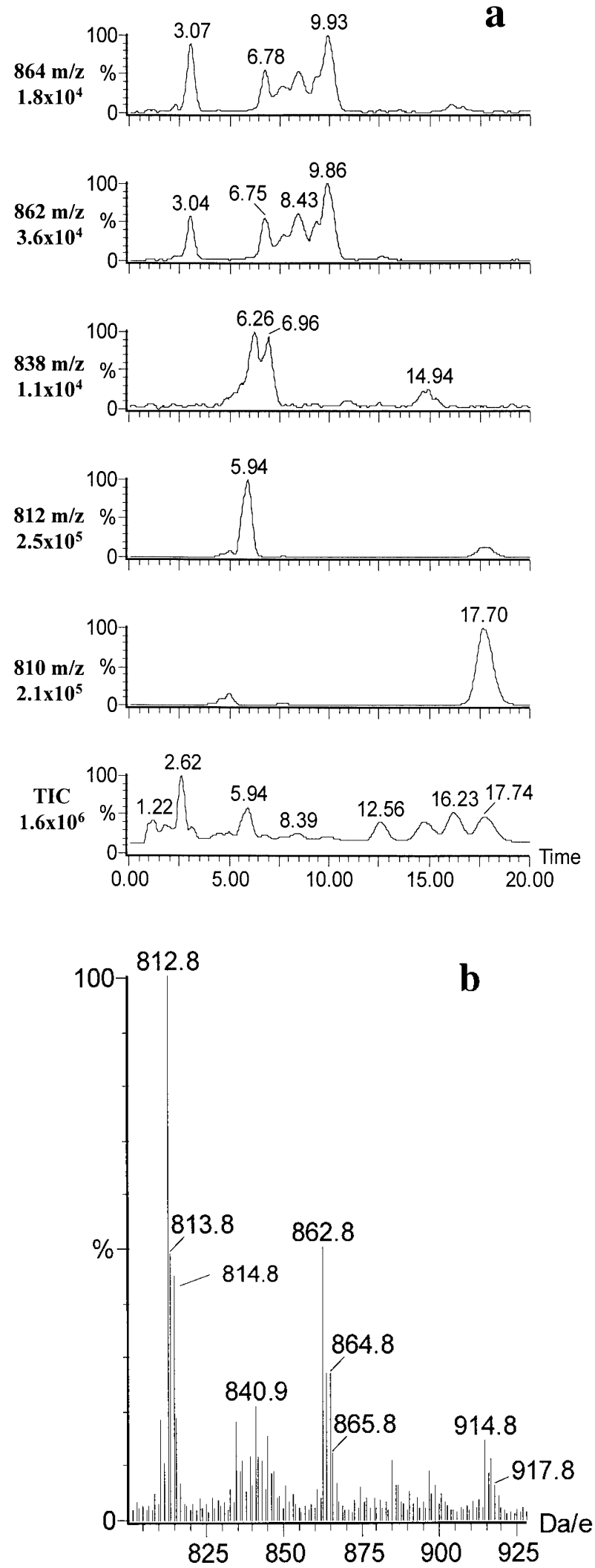

Figure 3 The detection of chlorohydrins in phospholipid vesicles

(a) TIC and RICs of phospholipid vesicles treated with $1.85 \mathrm{mM} \mathrm{HOCl}$ for $40 \mathrm{~min}$. The individual masses for the RICs and the ion current intensity corresponding to $100 \%$ on the vertical scale are shown next to the $y$-axis. The vertical axes are not linked and the retention time is in min. (b) The summed spectrum corresponding to the part of the chromatogram where the modified PCs eluted. The vertical axis is normalized to the largest peak in the spectrum. Da/e is equivalent to $\mathrm{m} / \mathrm{z}$. the phospholipid profiles of both cell types were still found to be quite resistant to changes caused by this oxidizing system. This can be seen in Figure 4, which shows the TIC and RIC at $818 \mathrm{~m} / z$ for extracts of control U937 cells and U937 cells treated with $1 \mathrm{M} \mathrm{t}$-BHP $+1 \mathrm{mM} \mathrm{Fe}{ }^{2+}$. There are no obvious changes in the native lipid signals that can be observed in the TIC in the treated cell sample, and the peaks due to modified lipids in the early part of the chromatogram are extremely weak. However, the RIC of $818 \mathrm{~m} / z$ clearly shows the appearance of an oxidized $\mathrm{PC}$ at $6.5 \mathrm{~min}$, assigned as the hydroperoxide of $\mathrm{C}_{36: 2}$ $\mathrm{PC}$ (e.g. stearoyl-linoleoyl PC), in the treated sample but not in the control sample. The signals at $\approx 16.5 \mathrm{~min}$ correspond to the presence of a very minor native lipid species, possibly $\mathrm{C}_{38: 0} \mathrm{PC}$. A small signal at $790 \mathrm{~m} / z$ was also observed at $\approx 5 \mathrm{~min}$ in the treated sample, but not in the control sample; this was assigned to a monohydroperoxide of $\mathrm{C}_{34: 2} \mathrm{PC}$ (e.g. palmitoyl-linoleoyl PC), which has a native mass of 758 (results not shown).

The HL60 cell line appeared to be slightly more susceptible to oxidative stress, as treatment with the same levels of oxidants gave clearer signals for peroxides from $\mathrm{C}_{34: 2} \mathrm{PC}$ and $\mathrm{C}_{36: 2} \mathrm{PC}$ (Figure 5). The RIC at $790 \mathrm{~m} / z$ shows the appearance in the treated sample of a very clear signal eluting at $5.44 \mathrm{~min}$, assigned as the monoperoxide of $\mathrm{C}_{34: 2} \mathrm{PC}$, which was absent in the control sample. A number of other components with this mass are also apparent in the chromatogram: the largest peak at $\approx 19 \mathrm{~min}$ is probably due to $\mathrm{C}_{36: 0} \mathrm{PC}$, present at very low concentrations in these cells; the other signals in the chromatogram between 7.85 and $13 \mathrm{~min}$ have not been assigned but are unlikely to be oxidized lipids as they are present in both samples. Similarly, there was evidence for the presence of a monoperoxide of $\mathrm{C}_{36: 2}$ $\mathrm{PC}$ in the t-BHP-treated sample, eluting at $6.42 \mathrm{~min}$ in the $818 \mathrm{~m} / z$ RIC. No comparable peak was present in the control sample, but a component with a mass of 818 found to elute later was assigned as $\mathrm{C}_{38: 0} \mathrm{PC}$; this is much smaller in the treated sample because the stronger signal at $6.42 \mathrm{~m} / z$ results in a higher scale on the $y$ axis. No bisperoxides were observed from either cell line, which probably reflects the low levels of fatty acids with more than two double bonds.

The effect of stressing U937 cells with $\mathrm{HOCl}$ was also investigated, as this is an important microbicidal oxidant released by activated phagocytes, and thought to be involved in inflammatory damage, for example in atherosclerosis. A comparison of the RICs at $812 \mathrm{~m} / z$ of the HOCl-treated sample and the control sample (Figure 6) shows clearly the formation of a modified lipid at $6.38 \mathrm{~min}$ in the HOCl-treated cells that is absent in control cells, which would correspond to the chlorohydrin of $\mathrm{C}_{34: 1} \mathrm{PC}$ $(760+52 \mathrm{~m} / z)$. Analysis of the spectrum from this region showed that the modified lipid at $812 \mathrm{~m} / \mathrm{z}$ was the major component and that the ${ }^{37} \mathrm{Cl}$ isotope at $814 \mathrm{~m} / \mathrm{z}$ was also present in the appropriate ratio. Signals at 784 and $786 \mathrm{~m} / z$ in the expected isotope ratio for a chlorohydrin were also observed, providing evidence of chlorohydrin formation from $\mathrm{C}_{32: 1} \mathrm{PC}$ (native mass 732). None of these signals were present in any significant quantities in the same region of the control sample.

\section{Dose-response and time course of hydroperoxide formation in U937 cells}

Having established by using high t-BHP concentrations that hydroperoxide formation from di-unsaturated phospholipids could be observed in oxidatively stressed cells, further treatments were carried out at lower concentrations of t-BHP and with varying incubation times, to provide more information on the response of the cells to oxidative stress and to determine the level of stress required to produce detectable peroxides. The left-hand 


\section{Table 1 Masses of native and modified PC species observed in this study using LC-MS}

The molecular masses of monochlorohydrin and hydroperoxide derivatives are given, and the adjacent columns show whether these modified lipids were observed in vesicles or in mammalian cells treated with either $\mathrm{HOCl}$ or $\mathrm{t}-\mathrm{BHP}+\mathrm{Fe}^{2+}$. A dash indicates where oxidative modifications would not be expected.

\begin{tabular}{llllll}
\hline PC assignment & Native $m / z$ & Chlorohydrin $m / z$ & Observed in & Hydroperoxide $m / z$ & Observed in \\
\hline$C_{30: 0}$ (e.g. myristoylpalmitoyl PC) & 706 & - & - & - & - \\
$C_{32: 1}$ (e.g. palmitoylpalmitoleoyl PC) & 732 & $784+786$ & Vesicles and cells & - & - \\
$C_{32: 0}$ (e.g. dipalmitoyl PC) & 734 & - & - & - & - \\
$C_{34: 2}$ (e.g. palmitoyl-linoleoyl PC) & 758 & $810+812$ & Vesicles only & 790 & Vesicles and cells \\
$C_{34: 1}$ (e.g. palmitoyoleoyl PC) & 760 & $812+814$ & Vesicles and cells & - & - \\
$C_{36: 2}$ (e.g. stearoyl-linoleoyl PC) & 786 & $838+840$ & Vesicles and cells & 818 & Vesicles and cells \\
$C_{38: 4}$ (e.g. stearoylarachidonoyl PC) & 810 & $862+864$ & Vesicles only & $842 / 874 / 906$ & Vesicles only
\end{tabular}
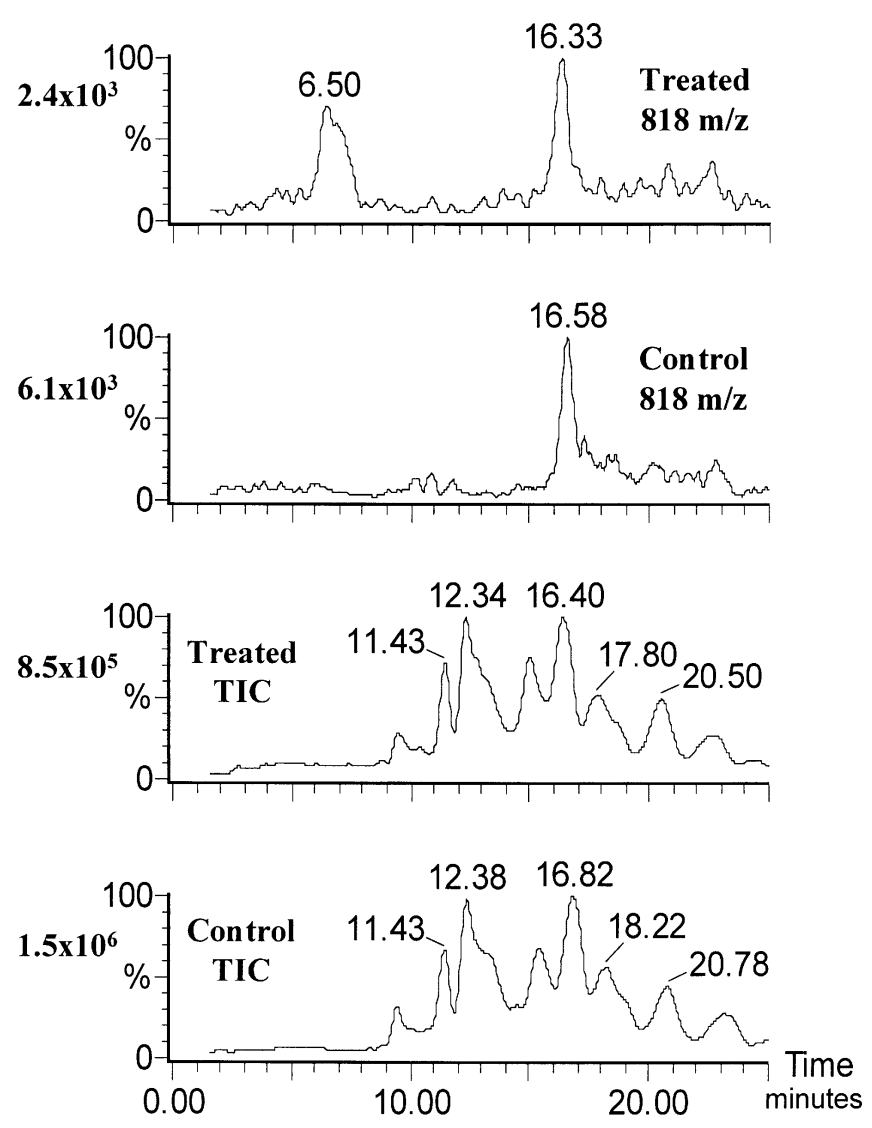

\section{Figure 4 LC-MS of U937 cells treated with $\mathrm{t}-\mathrm{BHP}$ and $\mathrm{Fe}^{2+}$}

The TICs and RICs at $818 \mathrm{~m} / z$ for extracts of control U937 cells and U937 cells treated with $1 \mathrm{M} \mathrm{t}-\mathrm{BHP}+1 \mathrm{mM} \mathrm{Fe}^{2+}$ for $30 \mathrm{~min}$. Following treatment the cells were washed, extracted in chloroform/methanol, and the lipid extracts were dried under nitrogen. The extracts were reconstituted with 1:5 chloroform/methanol (100 $\mu$ l total volume) before diluting further in LC solvent (71:5:7, methanol/hexane/0.1 M ammonium acetate). LC-MS was carried out as described for Figure $1.100 \%$ on the vertical axes corresponds to the ion current intensity indicated next to the $y$ axis of the chromatograms; the vertical axes are not linked.

panel of Figure 7 gives the RICs at $790 \mathrm{~m} / \mathrm{z}$ for cells treated with increasing t-BHP concentrations. It can be seen that the peak at $5.23 \mathrm{~min}$, corresponding to $\mathrm{C}_{34: 2} \mathrm{PC}$ hydroperoxide, is absent in the control treatment $(0 \mathrm{mM})$ but increases with increasing t$\mathrm{BHP}$ concentration. The hydroperoxide can be observed readily following treatment of the U937 cells with $50 \mathrm{mM}$ t-BHP for $30 \mathrm{~min}$, the lowest concentration tested. The area of the peaks corresponding to $\mathrm{C}_{34: 2}$ PC hydroperoxide and $\mathrm{C}_{36: 2}$ PC hydro-
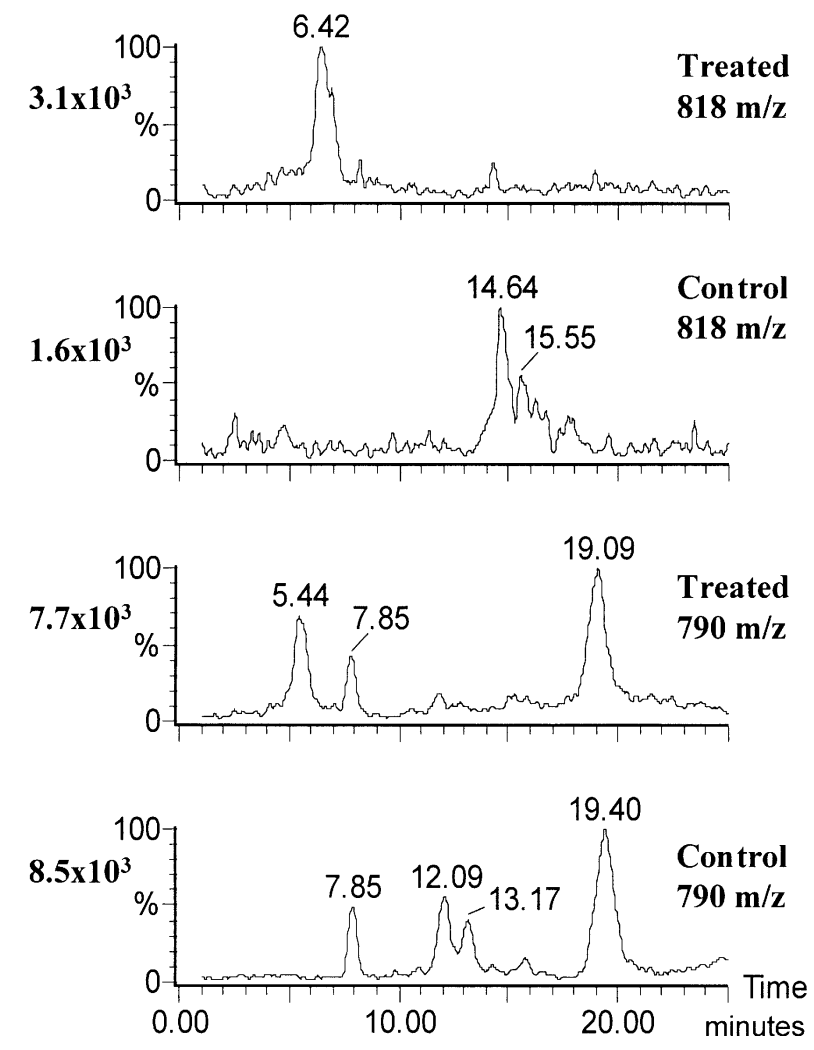

Figure 5 LC-MS of HL60 cells treated with t-BHP and $\mathrm{Fe}^{2+}$

The RICs at 790 and $818 \mathrm{~m} / \mathrm{z}$ for extracts of control and t-BHP-treated HL60 cells. All experimental procedures are as described for Figure 4. 100\% on the vertical axes corresponds to the ion current intensity indicated next to the $y$ axis of the chromatograms; the vertical axes are not linked.

peroxide (at $818 \mathrm{~m} / \mathrm{z}$ ) were determined and are shown in graphical format in Figure 7. The central panel shows that increasing the oxidant concentration resulted in an approximately linear increase in the peak area for both hydroperoxides over the concentration range tested. The effect of treatment with $0.5 \mathrm{M} \mathrm{t}$ BHP over a time course of $0-4 \mathrm{~h}$ was also investigated, and the calculated peak areas are shown in the right-hand panel of Figure 7. It was found that the levels of the hydroperoxides increased steadily over time, with detectable levels already present at the earliest time point of $15 \mathrm{~min}$. After $4 \mathrm{~h}$ the peaks corresponding to the hydroperoxides in the chromatograms were very large, with ion intensities of $7 \times 10^{3}(790 \mathrm{~m} / \mathrm{z})$ and $6 \times 10^{3}$ $(818 \mathrm{~m} / \mathrm{z})$. After $3 \mathrm{~h}$ treatment a peak corresponding to the bis- 

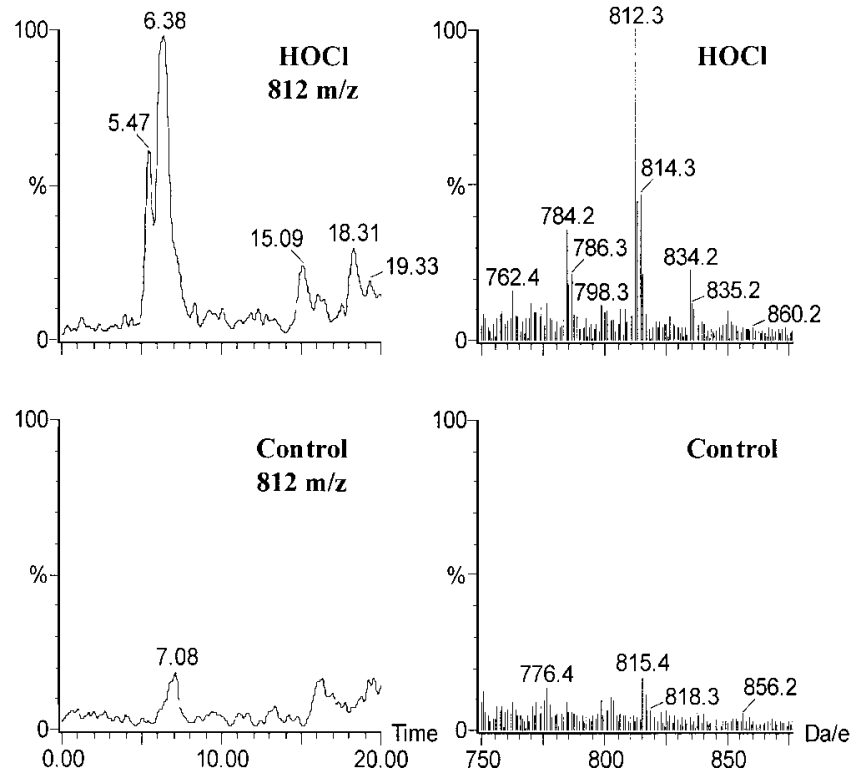

Figure 6 LC-MS of control and HOCl-treated U937 cells

The RICs at $812 \mathrm{~m} / \mathrm{z}$ and the summed spectra corresponding to the region where the modified PCs eluted ( $\approx 5-6.5 \mathrm{~min}$ ) are shown. The cells were treated with either PBS (control) or $3.5 \mathrm{mM} \mathrm{HOCl}$ for $30 \mathrm{~min}$, and were extracted and analysed as described for Figure 4. The vertical axes are linked both for the RICs and the spectra. Da/e is equivalent to $\mathrm{m} / \mathrm{z}$, and the retention times are in min.

hydroperoxide of $\mathrm{C}_{38: 4} \mathrm{PC}$ at $874 \mathrm{~m} / \mathrm{z}$ could also be detected, in addition to the monoperoxides observed. Thus, as expected, it can be seen that the levels of hydroperoxides present in the cells are dependent both on the severity and duration of the imposed oxidative insult.

\section{DISCUSSION}

This research has shown that specific intact oxidatively modified phospholipids can be detected in extracts of cells subjected to oxidative stress, by interfacing reversed-phase isocratic HPLC with a simple single quadrupole electrospray mass spectrometer using a suitable solvent system. This method allowed the detection of amounts of oxidatively modified lipid in the high femtomolar range, often corresponding to less than $0.5 \%$ of the corresponding native lipid, which was not possible by direct injection of the sample into the mass spectrometer. The improvement in performance compared with direct-injection ES-MS was due to the separation of oxidized and native phospholipids, which minimizes the occurrence of ion suppression and facilitates the observation of oxidized components in complex mixtures of lipids, rather than to an intrinsic increase in detection sensitivity. In particular, the advantage can be seen in the ability to resolve native and oxidized species of the same molecular mass, such as stearoyl-arachidonoyl PC and the monochlorohydrin of palmitoyl-linoleoyl PC, which both occur at $810 \mathrm{~m} / \mathrm{z}$. Compared with other commonly used techniques, such as GC-MS, this method has two important advantages. First, intact phospholipids can be observed, rather than the hydrolysed fatty acids, and therefore valuable information about the membrane-lipid composition is retained. Secondly, no prior hydrolysis or other chemical derivatization is required, making the technique rapid and straightforward to perform, and suitable for routine analysis of biological samples.
While the simplicity of this method is a significant advantage, it has the disadvantage compared with tandem MS that the species observed cannot be identified definitively by their fragmentation patterns. For example, a simple increase in molecular mass cannot be taken as absolute proof of the nature of a modification to a lipid. However, the loss of water [14] and early elution of these species, observed only in treated samples, is good evidence for their oxidative modification. Regarding the formation of chlorohydrins, the coincident appearance of an additional peak at +2 mass units at the expected ${ }^{35} \mathrm{Cl} /{ }^{37} \mathrm{Cl}$ isotope ratio provides strong support for their identification as a chlorohydrin. With respect to hydroperoxide formation, a mass increase of 32 in a lipid containing two double bonds can only reasonably be assigned as a peroxide; a bisepoxide or bishydroxide would have the same mass, but would be expected to derive from a bishydroperoxide, which is unlikely to occur when a single bisallylic hydrogen is present. Other oxidation products such as aldehydes would not result in the molecular mass increases observed. Especially in phospholipid vesicles, where the appearance of a modified species can be linked to the disappearance of the native lipid, the increases in mass are unequivocal. Similarly, a mass increase of 52 is difficult to account for other than by the addition of $\mathrm{HOCl}$ across a double bond to yield a lipid chlorohydrin.

In this study it has been shown that several specific intact phospholipid hydroperoxides and chlorohydrins occur in cells subjected to oxidative stress, and can be detected by LC-MS. Following free-radical-induced stress, the strongest signals observed were from monoperoxides of $\mathrm{C}_{34: 2}$ and $\mathrm{C}_{36: 2} \mathrm{PC}$, consistent with the fact that these were the most abundant diunsaturated lipids present in the cells. The only bishydroperoxide observed was from $\mathrm{C}_{38: 4}$ PC (stearoylarachidonoyl PC) after 3-4 h of treatment, but this is not surprising as the cultured cells contained relatively low levels of polyunsaturated fatty acyl chains within the PCs. As expected, the appearance of hydroperoxides was found to depend on both the severity and length of the oxidative treatment, increasing approximately linearly over the concentration range and time course tested. The monoperoxides could be detected clearly at the shortest time point (15 min), and at the lowest concentration of t-BHP $(50 \mathrm{mM})$ investigated. However, the native phospholipid profiles of the cells appeared to be quite resistant even to high levels of this oxidant system over relatively short time courses, with no obvious depletion of the unsaturated lipids according to the TIC trace.

With $\mathrm{HOCl}$, a much lower concentration was required to cause observable modification of the phospholipids, but at this concentration the native lipid profile was also not noticeably altered. The oxidative modifications observed in cells with this oxidant were only to mono-unsaturated PCs, specifically $\mathrm{C}_{32: 1}$ and $\mathrm{C}_{34: 1} \mathrm{PC}$, despite the fact that the cells contained a substantial proportion of di-unsaturated phospholipids and that chlorohydrins of such lipids were observed in the vesicle model system. However, it was also observed in the treatment of lipid vesicles that the monochlorohydrin of palmitoyloleoyl PC at $812 \mathrm{~m} / \mathrm{z}$ was the most abundant product, with an ion current almost an order of magnitude higher than the chlorohydrins of more unsaturated lipids. This could be due to better stability of a monochlorohydrin compared with more modified lipids, or simply because in a monounsaturated lipid the monochlorohydrin accumulates as the end product.

In both cell types and with both types of oxidizing system, the susceptibility of the cellular phospholipids to oxidative damage was lower than expected compared with effects on other cell types and tissues reported previously [17-19]. This may be due in part to the relatively small amount of polyunsaturated PCs 


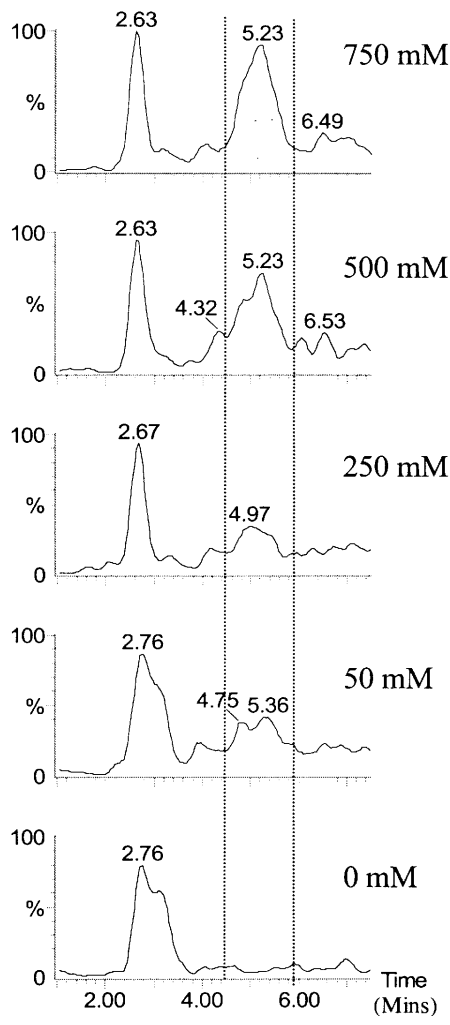

Dose Response
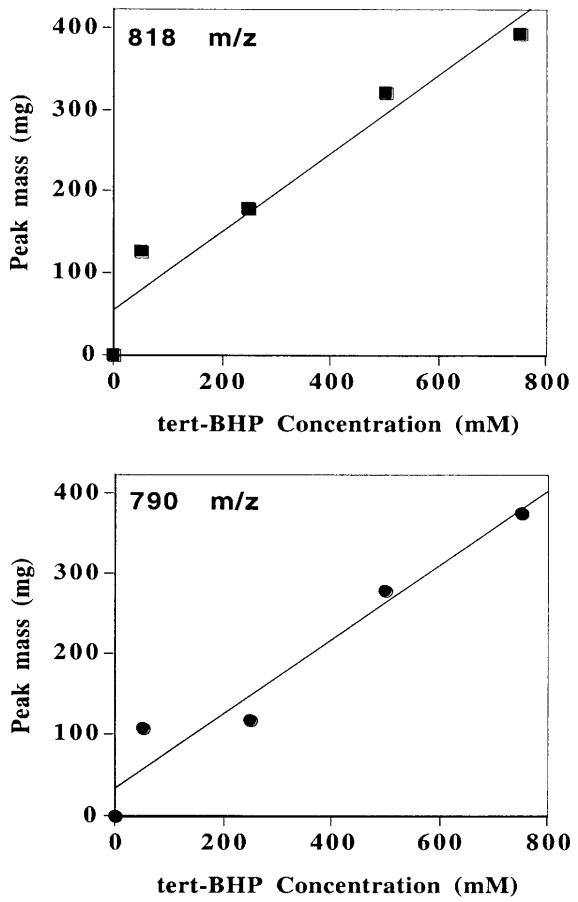

Time Course
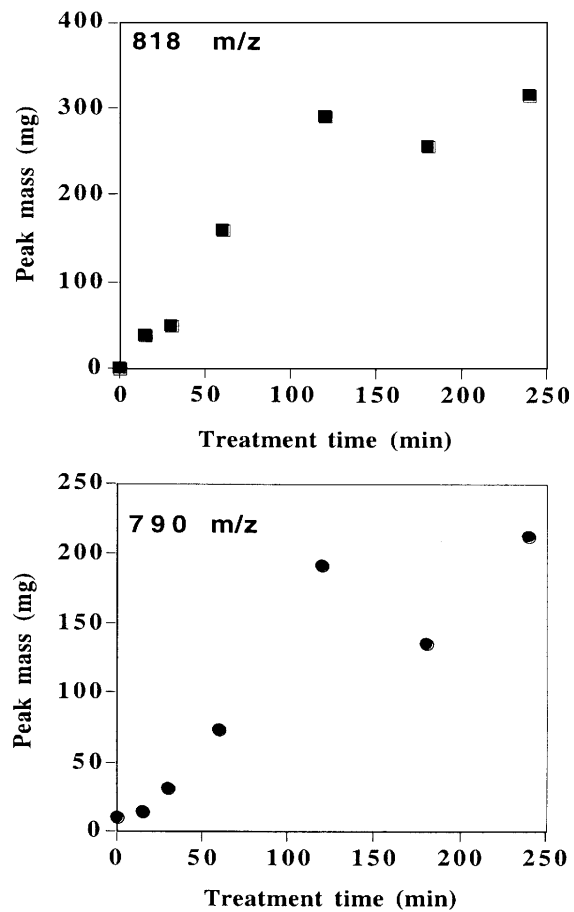

Figure 7 Effect of t-BHP concentration and treatment length on hydroperoxide levels

The left-hand panel shows the RICs at $790 \mathrm{~m} / \mathrm{z}$ of U937 cells treated with various concentrations of t-BHP and $1 \mathrm{mM} \mathrm{Fe}{ }^{2+}$. The intensities of the chromatograms are linked vertically with $100 \%$ corresponding to an ion intensity of $3.4 \times 10^{3}$, and the dotted lines either side of the peak at 5.23 min show the region of the chromatogram used for cutting and weighing to estimate the peak area. The central panel (dose-response) shows the relationship between t-BHP concentration and peak area for both $\mathrm{C}_{34.2} \mathrm{PC}$ hydroperoxide (790 $\mathrm{m} / \mathrm{z}$ ) and $\mathrm{C}_{36.2} \mathrm{PC}$ hydroperoxide (818 $\mathrm{m} / \mathrm{z}$ ) peaks. The peak areas were estimated by cutting and weighing the peaks in the printed chromatograms, hence the $y$ axis is given as peak mass in mg. The right-hand panel (time course) shows comparable data for the relationship between treatment time and hydroperoxide peak area.

present in these cells, which were not differentiated; it has been reported previously that differentiation of U937 cells with DMSO results in an increase in the level of arachidonate in PCs [25]. However, the cells appear to contain a high proportion of linoleic acid-containing PCs that can also be peroxidized readily in phospholipid vesicles, even if they are not as susceptible as arachidonic acid, so these would be expected to be depleted under the conditions used. The resistance of the cells may thus have more to do with preferential attack on other cellular components or selective antioxidant activity. It is perhaps pertinent that both the cell types used in these experiments are phagocytes, capable of generating oxidants for anti-microbial defence, and might therefore be expected to have enhanced antioxidant protection. It is also possible that oxidized fatty acids within phospholipids are selectively removed from the membrane to prevent physical disruption occurring, and that rapid turnover of membrane phospholipids prevents the accumulation of intact phospholipid oxidation products. In any case, membrane phospholipids seem to be highly protected against oxidative modification, especially by free-radical-generating systems. Thus the results described here give rise to interesting questions about the precise molecular events occurring at the plasma membrane during oxidative insult.

This research has shown that this combination of HPLC and ES-MS can yield a considerable amount of information about oxidative modifications to cellular lipids, detecting simultaneously and with comparable sensitivity a range of different oxidation products. This advance opens up the possibility of more detailed investigations of the molecular response of the cellular lipid pool during oxidative stress, in addition to the potential for its application to the study of oxidative damage in clinical situations.

C.M.S. would like to thank the Glaxo-Jack Endowment for financial support. A.J. and R.J.S. acknowledge support from the Austrian Science Fund (FWF) within the Joint Research Project 'Biomembranes' (project F711). L.Z. acknowledges support from the University of Bologna and MURST (Research project 'Free radicals and radical ions in chemical and biological processes').

\section{REFERENCES}

1 Halliwell, B. and Gutteridge, J. M. C. (1990) Role of free radicals and catalytic metal ions in human disease: an overview. Methods Enzymol. 186, 1-85

2 Carr, A. C., Vissers, M. C., Domigan, N. M. and Winterbourn, C. C. (1997) Modification of red cell membrane lipids by hypochlorous acid and haemolysis by preformed lipid chlorohydrins. Redox Rep. 3, 263-271

3 Luo, X. P., Yazdanpanah, M., Bhooi, N. and Lehotay, D. C. (1995) Determination of aldehydes and other lipid peroxidation products in biological samples by gas chromatography-mass spectrometry. Anal. Biochem. 228, 294-298

4 Van Kuijk, F. J. G. M., Thomas, D. W., Stephens, R. J. and Dratz, E. A. (1990) Gas chromatography-mass spectrometry of 4-hydroxynonenal in tissues. Methods Enzymol. 186, 399-406

5 Quinlan, G. J., Lamb, N. J., Evans, T. W. and Gutteridge, J. M. C. (1996) Plasma fatty acid changes and increased lipid peroxidation in patients with adult respiratory distress syndrome. Crit. Care Med. 24, 241-246 
6 Herold, M. and Spiteller, G. (1996) Enzymatic production of hydroperoxides of unsaturated fatty acids by injury of mammalian cells. Chem. Phys. Lipids $\mathbf{7 9 ,}$ $113-121$

7 Van Kuijk, F. J. G. M., Thomas, D. W., Stephens, R. J. and Dratz, E. A. (1990) Gas chromatography-mass spectrometry assays for lipid peroxides. Methods Enzymol. 186, 388-398

8 Matthews, W. R., Guido, D. M., Fisher, M. A. and Jaeschke, H. (1994) Lipid peroxidation as molecular mechanism of liver cell injury during reperfusion after ischemia. Free Radical Biol. Med. 16, 763-770

9 Nourooz-Zadeh, J., Gopaul, N. K., Barrow, S., Mallet, A. I. and Änggård, E. E. (1995) Analysis of F2-isoprostanes as indicators of non-enzymatic lipid peroxidation in vivo by gas chromatography-mass spectrometry: development of a solid-phase extraction procedure. J. Chromatogr. B 667, 199-208

10 Mann, M. (1990) Electrospray: its potential and limitations as an ionization method for biomolecules. Org. Mass Spectrom. 25, 575-587

11 Kerwin, J. L., Tuininga, A. R. and Ericsson, L. H. (1994) Identification of molecular species of glycerophospholipids and sphingomyelin using electrospray mass spectrometry. J. Lipid Res. 35, 1102-1114

12 Brügger, B., Erben, G., Sandhoff, R., Wieland, F. T. and Lehmann, W. D. (1997) Quantitative analysis of biological membrane lipids at the low picomole level by nanoelectrospray ionization tandem mass spectrometry. Proc. Natl. Acad. Sci. U.S.A. 94, 2339-2344

13 Waugh, R. J. and Murphy, R. C. (1996) Mass spectrometric analysis of four regioisomers of $\mathrm{F}$-2-isoprostanes formed by free radical oxidation of arachidonic acid. J. Am. Soc. Mass Spectrom. 7, 490-499

14 MacMillan, D. K. and Murphy, R. C. (1995) Analysis of lipid hydroperoxides and long chain conjugated keto acids by negative ion electrospray mass spectrometry. J. Am. Soc. Mass Spectrom. 6, 1190-1201

15 Carr, A. C., Winterbourn, C. C. and van den Berg, J. J. M. (1996) Peroxidase mediated bromination of unsaturated fatty acids to form bromohydrins. Arch. Biochem. Biophys. 327, 227-233

16 Khaselev, N. and Murphy, R. C. (1999) Susceptibility of plasmenyl glycerophosphoethanolamine lipids containing arachidonate to oxidative stress. Free Radical Biol. Med. 26, 275-284

Received 9 October 2000/8 January 2001; accepted 8 February 2001
17 Nakamura, T., Bratton, D. L. and Murphy, R. C. (1997) Analysis of epoxyeicosatetraenoic and monohydroxyeicosatetraenoic acids esterified to phospholipids in human red blood cells by electrospray tandem mass spectrometry. J. Mass Spectrom. 32, 888-896

18 Hall, L. M. and Murphy, R. C. (1998) Analysis of stable oxidized molecular species of glycerophospholipids following treatment of red blood cell ghosts with t-butylhydroperoxide. Anal. Biochem. 258, 184-194

19 Nakamura, T., Henson, P. M. and Murphy, R. C. (1998) Occurrence of oxidized metabolites of arachidonic acid esterified to phospholipids in murine lung tissue. Anal. Biochem. 262, 23-32

20 Spickett, C. M., Pitt, A. R. and Brown, A. J. (1998) Direct observation of lipid hydroperoxides in phospholipid vesicles by electrospray mass spectrometry. Free Radical Biol. Med. 25, 613-620

21 Watson, A. D., Leitinger, N., Navab, M., Faull, K. F., Hörkkö, S., Witztum, J. L., Palinski, W., Schwenke, D., Salomon, R. G., Sha, W. et al. (1997) Structural identification by mass spectrometry of oxidized phospholipids in minimally oxidized low density lipoprotein that induce monocyte/endothelial interactions and evidence for their presence in vivo. J. Biol. Chem. 272, 13597-13607

22 Watson, A. D., Subbanagounder, G., Welsbie, D. S., Faull, K. F., Navab, M., Jung, M. E., Fogelman, A. M. and Berliner, J. A. (1999) Structural identification of a novel pro-inflammatory epoxyisoprostane phospholipid in mildly oxidized low density lipoprotein. J. Biol. Chem. 274, 24787-24798

23 Bligh, E. G. and Dyer, W. J. (1959) Rapid method of total lipid extraction and purification. Can. J. Biochem. Physiol. 37, 911-917

24 Kim, H. Y., Wang, T. C. L. and Ma, Y. C. (1994) Liquid chromatography/mass spectrometry of phospholipids using electrospray ionisation. Anal. Chem. 66, 3977-3982

25 Hsu, F. F., Ma, Z. M., Wohltmann, M., Bohrer, A., Nowatzke, W., Ramanadham, S and Turk, J. (2000) Electrospray ionization/mass spectrometric analyses of human promonocytic U937 cell glycerophospholipids and evidence that differentiation is associated with membrane lipid composition changes that facilitate phospholipase A(2) activation. J. Biol. Chem. 275, 16579-16589

26 Jerlich, A., Pitt, A. R., Schaur, R. J. and Spickett, C. M. (2000) Pathways of phospholipid oxidation by $\mathrm{HOCl}$ in human LDL detected by LC-MS. Free Radical Biol. Med. 28, 673-682 